\title{
INCLUDING A NON-HOLONOMIC CONSTRAINT IN THE FSP (FULL SPACE PARAMETERIZATION) METHOD FOR MOBILE MANIPULATORS' MOTION PLANNING**
}

\author{
François G. Pin, Charles J. Hacker, Kathryn B. Gower, and Kristi A. Morgansen \\ Robotics and Process Systems Division \\ Oak Ridge National Laboratory \\ P.O. Box 2008 \\ Oak Ridge, TN 37831-6305
}
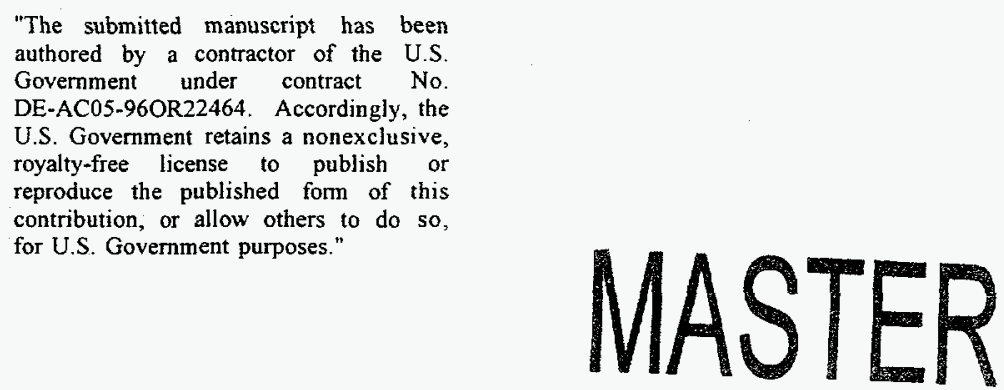

Submitted to: 1997 IEEE International Conference on Robotics and Automation, Albuquerque, New Mexico, April 21-27, 1997

Contact: Dr. François G. Pin, Oak Ridge National Laboratory, Building 7601, MS-6305, Oak Ridge, TN 37831-6305, Telephone: (423)574-6130, Fax: (423)574-4624, E-mail: pin@ornl.gov

*This research was supported in part by the U.S. Air Force Air Combat Command (ACC), the U.S. Air Force Munition Material Handling Equipment (MMHE) Focal Point, the U.S. Air Force Reliability and Maintainability Technology Insertion Program (PRAM-RAMTIP), the U.S. Department of Defense, Office of the Secretary of Defense (OSD), and the U.S. Advanced Project Research Agency (ARPA), under Interagency Agreement 2146-H055-A1 between the U.S. Air Force Materiel Command (AFMC) San Antonio Air Logistics Center, Robotics and Automation Center of Excellence (SA/ALC-RACE) and the Oak Ridge National Laboratory, managed by Lockheed Martin Energy Research Corp. for the U.S. Department of Energy under contract number DE-AC05-96OR22464; and in part by the DOE-sponsored Professional Internship Program (PIP) and the Science and Engineering Research Semester (SERS) Program administered by the Oak Ridge Institute for Science and Education. 


\section{DISClamigr}

Portions of this document may be illegible in electronic image products. Images are produced from the best available original document. 


\title{
INCLUDING A NON-HOLONOMIC CONSTRAINT IN THE FSP (FULL SPACE PARAMETERIZATION) METHOD FOR MOBILE MANIPULATORS' MOTION PLANNING
}

\author{
Contact: Dr. François G. Pin, Oak Ridge National Laboratory, P.O. Box 2008, Oak Ridge, \\ TN 37831-6305, Telephone: (423)574-5346, Fax: (423)574-4624, E-mail: pin@ornl.gov
}

\begin{abstract}
The efficient utilization of the motion capabilities of mobile manipulators, i.e., manipulators mounted on mobile platforms, requires the resolution of the kinematically redundant system formed by the addition of the degrees of freedom (d.o.f.) of the platform to those of the manipulator. At the velocity level, the linearized Jacobian equation for such a redundant system represents an underspecified system of algebraic equations, which can be subject to a set of constraints such as obstacles in the workspace and various limits on the joint motions. A method, which we named the FSP (Full Space Parameterization), has recently been developed to resolve such underspecified systems with constraints that may vary in time and in number during a single trajectory. The application of the method to motion planning problems with obstacle and joint limit avoidance was discussed in some of our previous work. In this paper, we present the treatment in the FSP of a non-holonomic constraint on the platform motion, and give corresponding analytical solutions for resolving the redundancy with a general optimization criterion. Comparative trajectories involving a 10 d.o.f. mobile manipulator testbed moving with and without a non-holonomic constraint for the platform motion, are presented to illustrate the use and efficiency of the FSP approach in motion planning problems for highly kinematically redundant and constrained systems.
\end{abstract}

Keywords: Mobile Manipulator, Redundancy Resolution, Non-Holonomic Constraint, Car-Like Robots, Full Space Parameterization, Motion Planning, Constrained Optimization

\section{SUMMARY}

Practical, outdoor mobile manipulators typically include a mobile platform which has car-like kinematics. Very few authors have addressed the problem of motion planning for mobile manipulators, and even fewer have considered this problem while including the non-holonomic constraint on the platform motion resulting from the car-like kinematic. This paper presents a method which analytically calculates motion solutions for the highly kinematically redundant mobile manipulators. The method is applicable either to systems with omnidirectional holonomic platforms or to systems including car-like platforms which exhibit a non-holonomic constraint. Example of trajectories are presented, some showing the typical "cusp points" (where velocity reversal occurs) of car-like motion. 


\title{
INCLUDING A NON-HOLONOMIC CONSTRAINT IN THE FSP (FULL SPACE PARAMETERIZATION) METHOD FOR MOBILE MANIPULATORS' MOTION PLANNING
}

\author{
François G. Pin, Charles J. Hacker, Kathryn B. Gower, and Kristi A. Morgansen \\ Robotics and Process Systems Division \\ Oak Ridge National Laboratory \\ P.O. Box 2008 \\ Oak Ridge, TN 37831-6305
}

\begin{abstract}
The efficient utilization of the motion capabilities of mobile manipulators, i.e., manipulators mounted on mobile platforms, requires the resolution of the kinematically redundant system formed by the addition of the degrees of freedom (d.o.f.) of the platform to those of the manipulator. At the velocity level, the linearized Jacobian equation for such a redundant system represents an underspecified system of algebraic equations, which can be subject to a set of constraints such as obstacles in the workspace and various limits on the joint motions. A method, which we named the FSP (Full Space Parameterization), has recently been developed to resolve such underspecified systems with constraints that may vary in time and in number during a single trajectory. The application of the method to motion planning problems with obstacle and joint limit avoidance was discussed in some of our previous work. In this paper, we present the treatment in the FSP of a non-holonomic constraint on the platform motion, and give corresponding analytical solutions for resolving the redundancy with a general optimization criterion. Comparative trajectories involving a 10 d.o.f. mobile manipulator testbed moving with and without a non-holonomic constraint for the platform motion, are presented to illustrate the use and efficiency of the FSP approach in motion planning problems for highly kinematically redundant and constrained systems.
\end{abstract}

\section{INTRODUCTION}

With the combined use of their mobility and manipulation capabilities, and their typically high degree of kinematic redundancy created by the addition of the platform d.o.f. to those of the manipulator, mobile manipulators can accomplish a great variety of tasks. Each one of these tasks is typically associated with a particular motion mode (platform only, manipulator only, combined motion), particular task requirements or optimization objectives (e.g., minimum time motion, optimal strength configuration, minimum spent energy, maximum dexterity, etc.) and particular constraints (e.g., obstacles, joint limits, non-holonomic constraint during platform motion, etc.) which may vary during a single trajectory. This great diversity of operational modes introduces additional complexity for mobile manipulator motion planning and control compared to fixed base manipulators and/or mobile robots without manipulators, in particular with respect to (1) the need to forecast specific configurations that are suitable for the system to switch from one operational mode to another, in a sequence of varied tasks, and (2) the need for a redundancy resolution method for planning the motion of the system between these "commutation" configurations.

In Refs. 1 and 2, we addressed the former need and introduced the concept of "commutation configurations" with a variety of approaches to calculate them.

With respect to the second need, several authors have previously addressed the problem of redundancy resolution, and Ref. 3 provides a good review of several approaches for applications to fixed based manipulators. In particular, the constrained problem has been investigated by several authors (e.g., see Refs. 4-7 for applications to obstacle and joint limit avoidance). All of these approaches use one of the two main techniques for resolution of underspecified systems of equation: constrained generalized inverse-based approaches or augmented task space methods with "extended Jacobians". In Ref. 9 we pointed out some of the shortcomings encountered when using either of these two general resolution approaches for application to real-time systems where constraints and/or task requirements may change widely and rapidly (e.g., at loop-rate and/or on a sensor-based basis) during a single trajectory. Among these shortcomings are the implicit task priority requirement of generalized inverse-based techniques, and the "artificial" algorithmic singularities that may be encountered with extended Jacobian and augmented task space approaches. More importantly, the variety of algorithms corresponding to each specific application of these techniques with a particular set of task requirements, optimization objectives and constraints would require a 
"library" of codes to be stored on-board the robot, which would be frequently switched to and from, to handle the expected diversity of operation modes of mobile manipulators.

In recent papers, ${ }^{9,10,11,12}$ we introduced a novel approach to the resolution of underspecified systems of algebraic equations subject to a variety of constraints and objective criteria. For robot control, the method can therefore be used for resolution of the velocity equation when constraints and task requirements vary rapidly and unpredictably with time during a single trajectory. The next section of this paper reviews the principles of this new approach, which we have named the Full Space Parameterization (FSP) approach, and recalls some of the analytical solutions developed for fixed based manipulator control, ${ }^{9}$ including those for obstacle and joint limit constraints. ${ }^{10}$ For applications to realistic mobile manipulators, a particular constraint which often restricts the motion of the platform must be considered. This constraint is of the non-holonomic type (e.g., see Refs. 13,14 , or 15 ), and very few authors have addressed its inclusion in the motion planning problem for mobile manipulators. ${ }^{16,17,18}$ In the remainder of the article, we present the treatment within the FSP framework of a non-holonomic constraint on the platform motion of mobile manipulators. Analytical solutions are derived and sample trajectories for a representative mobile manipulator system are presented to illustrate the analytical developments. The last section presents our conclusions and directions for future work.

\section{OVERVIEW OF THE FSP APPROACH}

For any mobile manipulator system, the forward kinematics can be reduced to the equation

$$
\bar{x}=\bar{x}_{p}+F\left(\bar{q}_{m}\right)
$$

where $\bar{x}$ and $\bar{x}_{p}$ are the $6 \times 1$ vectors of location and orientation of the mobile manipulator end-effector and of the platform reference frame with respect to the world coordinate system (task space), $\bar{q}_{m}$ is the $p \times 1$ vector of joint coordinates of the manipulator, and $F()$ is the manipulator kinematic transformation function. For loop-rate control, the desired end-effector motions in the task space are broken up into finite displacements of length $\Delta \bar{x}$. The relationship between the task space steps $\Delta \bar{x}$ and the mobile manipulator's configuration steps $\Delta \bar{q}^{T}=\left(\Delta \bar{q}_{m}, \Delta \bar{x}_{p}\right)$, where the upper $T$ sign denotes a transpose, is found by differentiating and linearizing Eq. (1):

$$
\Delta \bar{x}=\Delta \bar{x}_{p}+\frac{\partial F}{\partial \bar{q}_{m}} \Delta \bar{q}_{m}=J \Delta \bar{q}
$$

where $J$ is the linearized Jacobian for the entire mobile manipulator system over the current time step $\Delta t$.

For a redundant system, $J$ will have fewer rows $(n)$ than columns $(m=p+6)$, and the number of vectors $\Delta \bar{q}$ which satisfy Eq. (2) will typically be infinite. The FSP method has been specifically designed to optimally solve the inverse kinematics problem for redundant systems in the presence of applied constraints and behavioral criterion. In a previous paper, ${ }^{9}$ we showed that the entire space of solutions, $S$, of the unconstrained Eq. (2) could be parameterized as:

$$
S=\left\{\Delta \bar{q} \in \Re^{m}, \Delta \bar{q}\left(t_{1}, \ldots, t_{m-n+1}\right)=\sum_{i=1}^{m-n+1} t_{i} \bar{g}_{i}, \sum_{k=1}^{m-n+1} t_{k}=1\right\}
$$

where each of the linearly independent vectors $\bar{g}_{i}$ includes $m-n$ zero components and can be easily calculated from inversion of square $(n \times n)$ submatrices of $J$. The proof of existence and algorithms for the determination of these $m-n+1$ linearly independent solution vectors $\bar{g}_{i}$ can be found in Refs. 9, 11, and 12. In Ref. 9, it was also shown that the null space $\mathcal{N}$ of the mapping $J$ can be parameterized using the same $\bar{g}_{i}$ vectors as:

$$
\mathcal{N}=\left\{\Delta \bar{q} \in \Re^{m}, \Delta \bar{q}\left(t_{1}, \ldots, t_{m-n+1}\right)=\sum_{i=1}^{m-n+1} t_{i} \bar{g}_{i}, \sum_{k=1}^{m-n+1} t_{k}=0\right\}
$$

At each time step therefore, a calculation of the vectors $\bar{g}_{i}$ for Eq. (2) provides a parameterization of the entire spaces of solutions of the unconstrained system, be it for an end-effector motion or a motion in the null space. This phase of the FSP which is the most computational time consuming phase of the method, as shown in Refs. 11 and 12 , is therefore common to all time steps independently of their particular constraints and criteria. Then, with the entire spaces of solutions of Eq. (2) now parameterized, the calculation of the specific solution satisfying the particular task requirement and all the constraints of a time step is only the matter of a few code statements embodying the analytical expression of the corresponding solution parameters $t_{k}, k=1, m-n+1$. Practically, therefore, a wide variety of these parameter solutions, each corresponding to particular types of requirements and constraints, can be included in the code and selected as appropriate at each time step.

As shown in Refs. 9 and 10, analytical solutions for the parameters can be obtained from a Lagrangian-type constrained optimization. For example, consider optimization in the space defined by Eq. (3) of a general criterion 


$$
Q=\left\|\Delta \bar{Z}(\bar{q}, \Delta \bar{q})-\Delta \bar{Z}_{r}\right\|^{2}
$$

where $\Delta \bar{Z}$ is an operational vector function of the system's configuration and displacements expressed as:

$$
\Delta \bar{Z}=B(\bar{q}) \Delta \bar{q}
$$

and $\Delta \bar{Z}_{r}$ represents a given reference operational vector characterizing the state to be achieved by the system. Assume that, at the time step considered, the system is subject to a set of $r$ general constraints expressed as:

$$
\bar{\beta}^{j^{T}} \bar{t}=1 ; j=1, r
$$

a form to which many kinematic constraints (e.g., joint limits, obstacle avoidance, etc.) can be reduced, as discussed in detail in Ref. 10. Then the solution for the parameter set $\bar{t}^{T}=\left(t_{1}, t_{2}, \ldots, t_{m-n+1}\right)$ is:

$$
\bar{t}=-G^{-1}(\mu \bar{e}+B \bar{v}+\bar{H})
$$

where $B$ is a matrix whose columns are $\bar{\beta}^{i}$, and

$$
\begin{gathered}
\bar{H}, H_{k}=\Delta \bar{Z}_{r}^{T} B \bar{g}_{k} ; k=1, m-n+1 \\
G, G_{i j}=\bar{g}_{i}^{T} B^{T} B \bar{g}_{j} ; i, j=1, m-n+1 \\
\bar{e}, e_{i}=1 ; i=1, m-n+1 \\
a=\bar{e}^{T} G^{-1} \bar{e} \\
f=\bar{e}^{T} G^{-1} \bar{H} \\
\bar{b}, b_{i}=\bar{e}^{T} G^{-1} \bar{\beta}^{i}=\bar{\beta}^{i} G^{-1} \bar{e} ; i=1, r \\
\bar{d}, d_{i}=1+\bar{\beta}^{i} G^{-1} \bar{H} ; i=1, r \\
A, A_{i j}=b_{i} b_{j}-a \bar{\beta}^{i T} G^{-1} \bar{\beta}^{j} ; i=1, r ; j=1, r \\
\bar{v}=A^{-1}(a \bar{d}-\bar{b}(1+f)) \\
\mu=-\left(\bar{v}^{T} \bar{b}+1+f\right) / a
\end{gathered}
$$

In a very similar manner, if a constrained solution in the null space of Eq. (4) is desired, the solution for $\bar{t}$ is also given by Eqs. (8)-(18) except for the factor $1+f$ in Eqs. (17) and (18) replaced by $f$.

The approach for calculating the coefficient vectors $\bar{\beta}^{i}$ expressing the various constraints has been described in detail in Ref. 10. In particular, the cases of joint limit and obstacle avoidance, and bounded joint accelerations were presented in Refs. 10 and 19 , respectively. Essentially, the scheme which we used in Ref. 10 to implement obstacle avoidance considers that the mobile manipulator system is surrounded by a "safety envelop" or "danger zone" of thickness $D$. Whenever intersection of this zone with an obstacle occurs (the detection can be sensor-based), then a constraint is set, specifying that the closest point $X_{j}$ of the mobile manipulator to the obstacle must move away from the obstacle by a distance $L$ (the "intrusion" or "push away" distance). The expression for the vector $\bar{\beta}$ representing this constraint was derived in Ref. 10 as:

$$
\bar{\beta}, \beta_{k}=\sum_{i=1}^{3} \sum_{j=1}^{m}\left(J_{i j}^{X} g_{k_{j}} n_{i}\right) / L ; k=1, m-n+1
$$

where $J^{X}$ is the $3 \times m$ Jacobian matrix for the position displacement of the point $X_{j}$, and $g_{k_{j}}$ and $n_{j}$ represent the components of the vector $\bar{g}_{k}$ and of the normal $\bar{n}$ to the obstacle surface toward $X_{j}$, respectively.

In a similar fashion, if any joint, $i$, of the manipulator is approaching one of its limits, and requires an angle displacement, $d$, to return outside of its "danger zone" (angles within a $\theta_{\text {dang }}$ range of the limit), the $\bar{\beta}$ vector corresponding to this constraint can be expressed as:

$$
\bar{\beta}, \beta_{k}=g_{k_{i}} / d ; k=1, m-n+1
$$

The reader is referred to Ref. 10 for the details of these derivations.

\section{NON-HOLONOMIC CONSTRAINT ON THE PLATFORM MOTION}

Figure 1 shows an example of a mobile platform with a non-holonomic constraint. In what follows, we will assume that the platform reference frame is moving in a plane and that the World reference frame is chosen such that only three components of $\Delta \bar{x}_{p}$ are non-zero (i.e., tilt, roll, and elevation of the platform remain constant). The point denoted $P$ represents the reference frame of the platform with coordinate $x_{p}$ and $y_{p}$ with respect to the world coordinate frame $(O, \vec{i}, \vec{j})$ and with its first axis vector, $\vec{i}^{\prime}$, oriented along the centerline of the vehicle at an angle $\theta_{p}$ with $\vec{i}$. Points $M$ and $N$ respectively represent the middle of the rear axle with non-steerable wheels and the middle of the front axle with steerable wheel(s). $L$ and $W$ denote the distance between points $M$ 
and $P$, and points $M$ and $N$, respectively. The non-holonomic constraint can be derived by expressing that the velocity $\vec{V}_{M}\left(\dot{x}_{m}, \dot{y}_{m}\right)$ of point $M$ is always along the main axis of the vehicle:

$$
-\dot{x}_{m} \sin \theta_{p}+\dot{y}_{m} \cos \theta_{p}=0
$$

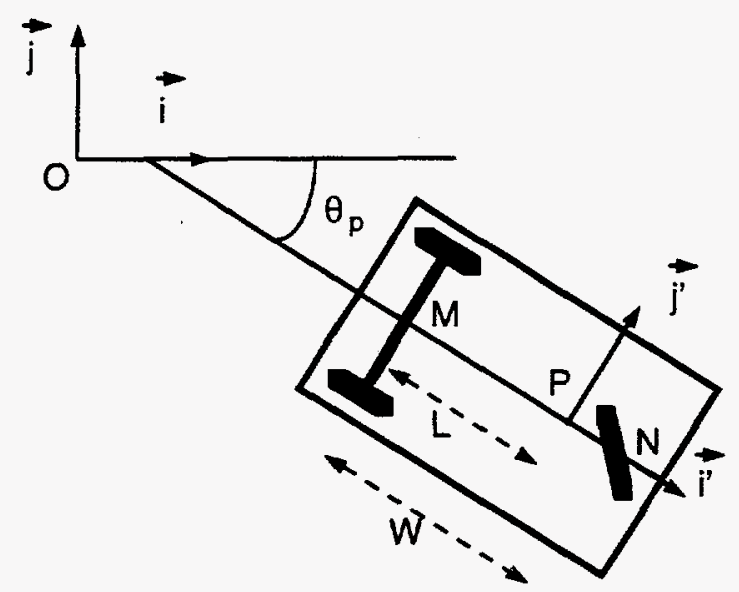

Fig. 1. Schematic of a non-holonomic platform.

Since the velocity $\vec{V}_{P}\left(\dot{x}_{p}, \dot{y}_{p}\right)$ of the platform point of reference $P$ is

$$
\vec{V}_{P}=\vec{V}_{M}+L \dot{\theta}_{p} \vec{j}^{\prime}
$$

or

$$
\left\{\begin{array}{l}
\dot{x}_{p}=\dot{x}_{m}-L \dot{\theta} \sin \theta_{p} \\
\dot{y}_{p}=\dot{y}_{m}+L \dot{\theta} \cos \theta_{p}
\end{array}\right.
$$

then the constraint of Eq. (23) can be written in terms of the platform configuration variables as:

$$
-\dot{x}_{p} \sin \theta_{p}+\dot{y}_{p} \cos \theta_{p}-L \dot{\theta}_{p}=0
$$

or, in linearized form over the discretized time step $\Delta t$ :

$$
-\Delta x_{p} \sin \theta_{p}+\Delta y_{p} \cos \theta_{p}-L \Delta \theta_{p}=0
$$

Note that this constraint is intrinsically due to the motion characteristics of wheels and is valid for platforms with two axles (one with steerable wheels) such as cars or carts similar to the one sketched in Fig. 1 (e.g., see Refs. 13, 14 , or 15 and references therein), as well as for platforms with directional control provided through independent driving of the two parallel wheels, with casters on the other axles for stability.
The constraint of Eq. (21) or Eq. (24) is of the form $C(\bar{q}, \dot{q})=0$ which binds the configuration variables and their derivatives. It is not integrable and therefore is properly a non-holonomic constraint which constrains the space of achievable velocities without constraining the space of achievable configurations.

As mentioned previously, the displacement vector $\Delta \bar{q}$ for the mobile manipulator has been constructed such that its first $m$ components refer to the manipulator. We can select its next three components to refer to the non-zero displacement components for the platform, i.e., $\Delta q_{m+1}=\Delta x_{p}, \Delta q_{m+2}=\Delta y_{p}$, and $\Delta q_{m+3}=\Delta \theta_{p}$. Equation (25) can thus be written as:

$$
-\Delta q_{m+1} \sin \left(q_{m+3}\right)+\Delta q_{m+2} \cos \left(q_{m+3}\right)-\Delta q_{m+3} L=0
$$

and since

$$
\Delta \bar{q}=\sum_{i=1}^{m-n+1} t_{i} \bar{g}_{i}
$$

the non-holonomic constraint can be written in terms of the parameters $t_{i}, i=1, m-n+1$, as:

$$
\sum_{i=1}^{m-n+1} \alpha_{i} t_{i}=0=\bar{\alpha}^{T} \bar{t}
$$

where $\bar{\alpha}$ is an $((m-n+1) \times 1)$ vector with components $\alpha$, given by:

$$
\alpha_{i}=-\sin \left(q_{m+3}\right) g_{i_{m+1}}+\cos \left(q_{m+3}\right) g_{i_{m+2}}-L g_{i_{m+3}}
$$

and $g_{i k}$ represents the $k$ th component of the vector $\bar{g}_{i}$.

With the non-holonomic constraint expressed in terms of the sought parameters $t_{i}, i=1, m-n+1$, it can be added to the constrained optimization procedure which, with the same framework and definitions used in Eqs. (3) through (18), leads to the following Lagrangian:

$$
\begin{aligned}
L\left(t_{i}, \mu, v_{j}, \eta\right)=Q\left(t_{i}\right) & +\mu\left(\sum_{i=1}^{m-n+1} t_{i}-1\right)+\sum_{j=1}^{r} v_{j} C^{j}\left(t_{i}\right) \\
& +\eta\left(\sum_{i=1}^{m-n+1} \alpha_{i} t_{i}\right) .
\end{aligned}
$$

The optimality conditions $\left(\frac{\partial \mathcal{L}}{\partial t_{i}}=0 ; i=1, m-n+1\right.$; $\left.\frac{\partial L}{\partial \mu}=0 ; \frac{\partial L}{v_{j}}=0 ; j=1, r ; \frac{\partial L}{\partial \eta}=0\right)$ become: 


$$
\left\{\begin{array}{l}
G \bar{t}+\bar{H}+\mu \bar{e}+\sum_{i=1}^{r} v_{i} \bar{\beta}^{i}+\eta \bar{\alpha}=\bar{o} \\
\bar{e}^{T} \bar{t}=1 \\
\bar{\alpha}^{T} \bar{t}=0 \\
\bar{\beta}^{j^{T}} \bar{t}=1, \quad j=1, r
\end{array}\right.
$$

and the solution to the system is:

$$
\begin{gathered}
\bar{t}=-G^{-1}(\bar{H}+\mu \bar{e}+B \bar{v}+\eta \bar{\alpha}) \\
\mu=-\left(1+f+\bar{v}^{T} \bar{b}+\eta \ell\right) / a \\
\eta=\left[a k-\ell(1+f)-\bar{v}^{T}(\ell \bar{b}-a \bar{c})\right] /\left(\ell^{2}-s a\right) \\
\bar{v}=A^{-1}\left[(\ell k-s(1+f)) \bar{b}-(a k-\ell(1+f)) \bar{c}-\left(\ell^{2}-s a\right) \bar{d}\right]
\end{gathered}
$$

where $a, f$, the vectors $\bar{b}, \bar{d}, \bar{e}$, and $\bar{H}$, and the matrices $G$ and $B$ have been previously defined, and

$$
\begin{gathered}
k=\bar{\alpha}^{T} G^{-1} \bar{H} \\
\ell=\bar{e}^{T} G^{-1} \bar{\alpha}=\bar{\alpha}^{T} G^{-1} \bar{e} \\
s=\bar{\alpha}^{T} G^{-1} \bar{\alpha} \\
\bar{c}, c_{i}=\bar{\beta}^{i^{T}} G^{-1} \bar{\alpha}=\bar{\alpha}^{T} G^{-1} \bar{\beta}^{i} \\
A, A_{i j}=b_{i}\left(s b_{j}-\ell c_{j}\right)+c_{i}\left(a c_{j}-\ell b_{j}\right)+\left(\ell^{2}-s a\right) \bar{\beta}^{i} G^{-1} \bar{\beta}^{j}
\end{gathered}
$$

When considering motion in the null space of Eq. (4), the expression of the solution is exactly as written in Eqs. (32) through (40), except for the factor $(1+f)$ being written as $f$ in Eqs. (33) to (35).

\section{SAMPLE RESULTS}

The solution of Eq. (29) and Eqs. (32) through (40) was implemented and tested using several of our mobile manipulator testbeds that incorporate non-holonomic constraints. With our HERMIES-III robot, ${ }^{20}$ some comparative experiments could be performed, and Figs. 2 and 3 illustrate the significant differences between the motion of the system with and without the non-holonomic constraint on the platform motion. All motions shown in these two figures are for $\Delta \bar{Z}_{r}=\overline{0}$ and

with $B$ as the identity matrix (see Eqs. (5) and (6)). In both figures, the view is taken from above the system to better visualize the motion of the platform. The configuration of the system is displayed every 20 time steps only, to ease the visualization of the trajectory. In all cases, the 3-D end-effector trajectory is specified in both position and orientation $(n=6)$, leaving the 10 d.o.f. system with 4 degrees of redundancy (d.o.r.).

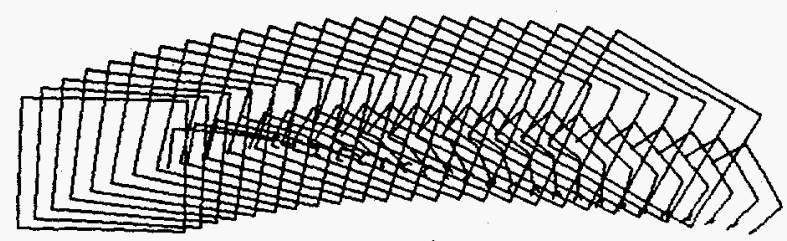

(a)

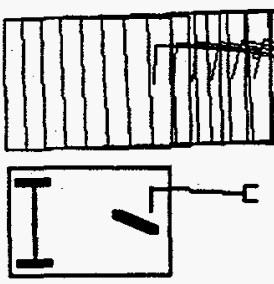

(b)

Fig. 2. Sample trajectory \#1 with (a) an omnidirectional platform, and (b) a non-holonomic constraint on the platform motion.
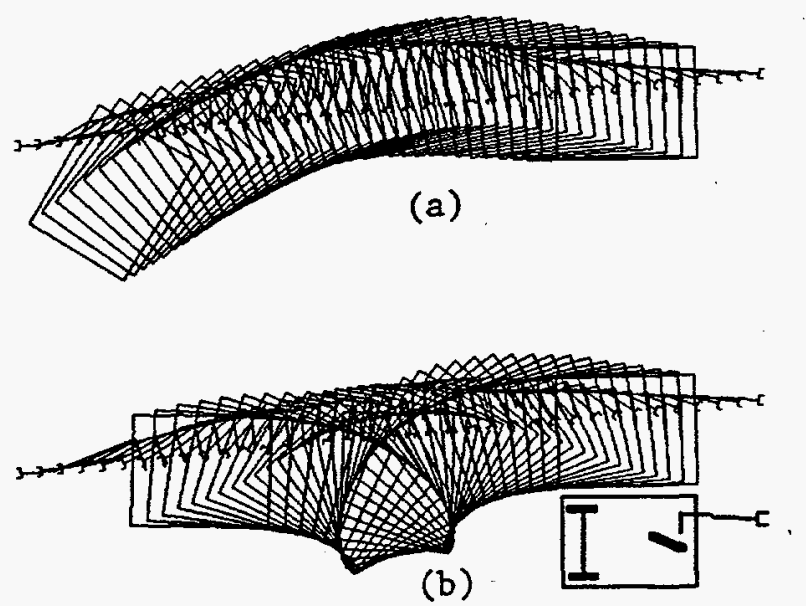

Fig. 3. Sample trajectory \#2 with (a) an omnidirectional platform, and (b) a non-holonomic constraint on the platform motion.

In each figure, the two motions of the system are for the same end-effector trajectory with (a) no constraint on the platform motion, i.e., utilizing the omnidirectionality of the HERMIES-III platform, ${ }^{20}$ and (b) a non-holonomic constraint on the platform motion, corresponding to the cart-like wheel kinematic illustrated in each figure by the 
little sketch showing the initial configuration of the system. In Fig. 2, the motion is from the left to the right of the figure with the platform moving "forward" (the axle with the non-steerable wheels is assumed to be the "rear" of the vehicle). In Fig. 2(a), the rear corners of the platform clearly illustrate omnidirectional motion with "sideways" displacement. In Fig. 2(b) the rear of the platform clearly "follows" the front of the vehicle in a "car-like" fashion.

In Fig. 3, the motion is from the right to the left of the figure, with the platform initially moving "backward." The end-effector is requested to follow a straight line trajectory passing above the initial location of the platform, while also yawing a total of $180^{\circ}$ over the entire trajectory. In Fig. 3(a) the omnidirectionality of the platform is clearly apparent through the "sideways" motion of the rear comers of the vehicle (e.g., see the left-hand side of the trajectory). In Fig. 3(b), while the non-holonomic platform initially moves "backward," the front of the vehicle, where the base of the manipulator is connected, is steering left (toward the top of the page) to try to accommodate for the displacement and yawing of the end-effector. This progressively makes the rear of the vehicle turn right (toward the bottom of the page) until a "cusp point" is reached where the platform reverses its overall motion direction, thereby moving "forward" to finish the trajectory.

The non-holonomic "car-like" motion is clearly illustrated in this figure through the motion of the rear of the platform, with no "sideways slippage" including at, and in the neighborhood of, the cusp point. Inspection of the detailed data file for this trajectory shows smooth and continuous variations of all the variables in the system, including at and in the neighborhood of the cusp point. Inspection of the data files for these and all other trajectories for all other systems experimented with, also shows that the non-holonomic constraint of Eq. (27) is always verified at each time step, with the absolute value for the left-hand side of the equation always less then $5 \times 10^{-5}$ (maximum value encountered), a value which is well within the expected errors due to numerical truncation and the linearization over the discretized time steps.

\section{CONCLUSION}

An approach to the motion planning of mobile manipulators including a non-holonomic constraint on the platform motion has been presented. This approach is based on the use of the FSP method to resolve the underspecified system of velocity equations with constraints. Analytical solutions have been derived and sample trajectories for holonomic and non-holonomic motions of one of our mobile manipulator testbeds have been presented to illustrate the use of the FSP approach and provide comparative results in these particular cases.

\section{ACKNOWLEDGMENT}

This research was supported in part by the U.S. Air Force Air Combat Command (ACC), the U.S. Air Force Munition Material Handling Equipment (MMHE) Focal Point, the U.S. Air Force Reliability and Maintainability Technology Insertion Program (PRAM-RAMTIP), the U.S. Department of Defense, Office of the Secretary of Defense (OSD), and the U.S. Advanced Project Research Agency (ARPA), under Interagency Agreement 2146-H055-Al between the U.S. Air Force Materiel Command (AFMC) San Antonio Air Logistics Center, Robotics and Automation Center of Excellence (SA/ALC-RACE) and the Oak Ridge National Laboratory, managed by Lockheed Martin Energy Research Corp. for the U.S. Department of Energy under contract number DE-AC05-96OR22464; and in part by the DOE-sponsored Professional Internship Program (for the second and fourth authors) and the Science and Engineering Research Semester (SERS) Program (for the third author) administered by the Oak Ridge Institute for Science and Education.

\section{REFERENCES}

1. F. G. Pin and J.-C. Culioli, "Optimal Positioning of Combined Mobile Platform-Manipulator Systems for Material Handling Tasks," J. Intell. and Rob. Syst., 6(2-3), 165-182, 1992.

2. F. G. Pin, J.-C. Culioli, and D. B. Reister, "Using Minimax Approaches to Plan Optimal Task Commutation Configurations for Combined Mobile Platform-Manipulator Systems," IEEE Trans. Rob. and Autom., 10(1), 44-54, 1994.

3. B. Siciliano, "Kinematic Control of Redundant Robot Manipulators: A Tutorial," J. Intell. Rob. Syst., 3, 201-212, 1990.

4. A. Liégeois, "Automatic Supervisory Control of the Configuration and Behavior of Multibody Mechanisms," IEEE Trans. Syst., Man, Cyber., 7, 868-871, 1977.

5. A. A. Maciejewski and C. A. Klein, "Obstacle Avoidance for Kinematically Redundant Manipulators in Dynamically Varying Environments," Int. J. Rob. Res., 4(3), 109-117, 1985.

6. R. Colbaugh, H. Seraji, and K. Glass, "Obstacle Avoidance for Redundant Robots Using Configuration Control," J. Rob. Sys., 6(6), 721-744, 1989.

7. P. Chiacchio et al., "Closed-Loop Inverse Kinematics Schemes for Constrained Redundant Manipulators with Task Space Augmentation and Task Priority Strategy," Int. J. Rob. Res., 10, 410-425, 1991.

8. J. Baillieul, "Kinematic Programming Alternatives for Redundant Manipulators," Proc. IEEE Int. Conf. Rob. Autom., St. Louis, Missouri, March 25-28, 1985, pp. 722-728. 
9. F. G. Pin et al. "A New Solution Method for the Inverse Kinematic Joint Velocity Calculations of Redundant Manipulators," Proc. IEEE Int. Conf. Rob. Autom., San Diego, California, May 8-13, 1994, pp. 96-102.

10. F. G. Pin and F. A. Tulloch, "Resolving Kinematic Redundancy with Constraints Using the FSP (Full Space Parameterization) Approach," Proc. IEEE Int. Conf. Rob. Autom., Minneapolis, Minnesota, April 22-28, 1996, pp. 468-473.

11. K. A. Morgansen and F. G. Pin, "Enhanced Code for the Full Space Parameterization Approach to Solving Underspecified Systems of Algebraic Equations," Oak Ridge National Laboratory Technical Report No. ORNL/TM-12816, 1995.

12. G. A. Fries, C. J. Hacker, and F. G. Pin, "FSP (Full Space Parameterization), Version 2.0" Oak Ridge National Laboratory Technical Report No. ORNL/TM-13021, 1995.

13. J. C. Latombe, Robot Motion Planning, Kluwer Academic Publishers, Boston, 1991.

14. H. A. Vasseur, F. G. Pin, and J. R. Taylor, "Navigation of Car-Like Mobile Robots in Obstructed Environments Using Convex Polygonal Cells," Rob. Auton. Syst., 10(2-3), 133-146, 1992.
15. J. P. Laumond, "Finding Collision-Free Smooth Trajectories for a Non-Holonomic Mobile Robot," Proc. 10th Int. Joint Conf. Art. Intell., Milan, Italy, 1987, pp. 1120-1123.

16. H. Seraji, "Configuration Control of Rover-Mounted Manipulators," Proc. IEEE Int. Conf. Rob. Autom., Nagaya, Japan, May 21-27, 1995, pp. 2261-2266.

17. Y. Yamamoto and X. Yun, "Control of Mobile Manipulators Following a Moving Surface," Proc. IEEE Int. Conf. Rob. Autom., Atlanta, Georgia, May 2-6, 1993, Vol. 3, pp. 1-6.

18. C. C. Wang and V. Kumar, "Velocity Control of Mobile Manipulators," Proc. IEEE Int. Conf. Rob. Autom., Atlanta, Georgia, May 2-6, 1993, Vol. 2, pp. 713-718.

19. K. A. Morgansen and F. G. Pin, "Impact Mitigation Using Kinematic Constraints and the Full Space Parameterization," Proc. IEEE Int. Conf. Rob. Autom., Minneapolis, Minnesota, April 22-28, 1996, pp. 1897-1902.

20. F. G. Pin et al., "Autonomous Mobile Robot Research Using the HERMIES-III Robot" Proc. IROS '89, The Int. Workshop Intell. Rob. Syst., Tsukuba, Japan, Sept. 4-6, 1989, pp. 251-256.

\section{DISCLAIMER}

This report was prepared as an account of work sponsored by an agency of the United States Government. Neither the United States Gnvernment nor any agency thereof, nor any of their employees, makes any warranty, express or implied, or assumes any legal liability or responsibility for the accuracy, completeness, or usefulness of any information, apparatus, product, or process disclosed, or represents that its use would not infringe privately owned rights. Reference herein to any specific commercial product, process, or service by trade name, trademark, manufacturer, or otherwise does not necessarily constitute or imply its endorsement, recommendation, or favoring by the United States Government or any agency thereof. The views and opinions of authors expressed herein do not necessarily state or reflect those of the United States Government or any agency thereof. 